\title{
Estudo comparativo da determinação experimental das constantes de sedimentabilidade de lodos ativados
}

\author{
Experimental determination comparative study of \\ activated sludge settling constants
}

Data de entrada: $12 / 10 / 2016$

Data de aprovação: $22 / 05 / 2017$

Luiz Guilherme Abreu de Paula*/Adrianus Cornelius van Haandel/ Heraldo Antunes Silva Filho/ Geilza Alves Porto DOI: 10.4322/dae.2018.006

\section{Resumo}

Realizou-se um estudo comparativo para avaliar os métodos estático e dinâmico de determinação das constantes $\mathrm{k} \mathrm{e} \mathrm{v}_{0}$ e a validade da Equação de Vesilind como parâmetro para caracterizar a sedimentabilidade de sistemas de lodo ativado. A investigação foi realizada com o lodo produzido por 3 diferentes sistemas de lodo ativado, que foram operados por um período de 155 dias. Os dados para a análise de sedimentabilidade para determinar as constantes de Vesilind $\mathrm{k}$ e $\mathrm{v}_{0}$ foram obtidas com os métodos estático e dinâmico, visando sua comparação. Os resultados experimentais mostraram que os dois métodos dão conclusões equivalentes sobre as constantes de Vesilind, uma vez que não houve diferença significativa entre os seus valores médios. A validade da equação de Vesilind foi confirmada tanto para os lodos gerados em sistema de fluxo contínuo (UCT) como em sistemas RBS e determinado pelos métodos dinâmico e estático. Palavras-chave: Sedimentabilidade. Equação de Vesilind. Lodo Ativado.

\section{Abstract}

We carried out a comparative study to evaluate the steady and dynamic determination methods for $k$ and $v_{0}$ constants and the validity of Vesilind equation as a parameter for characterizing the settling of sludge activated systems. The investigation was performed with sludge obtained from three sludge activated systems operated for 155 days. The steady method has been performed according to White (2004) and the dynamic method according to Leitão (2004). The experimental results showed that the two methods gave equivalent conclusions, once there was not a significant difference between their average values. The Vesilind Equation reliability was verified for sludges produced in a continuous flow system (UCT) and RBS systems, both measured by steady and dynamic methods. Keywords: Settling. Vesilind Equation. Sludge Activated.

\footnotetext{
Luiz Guilherme Abreu de Paula - Mestre em Engenharia Civil e Ambiental pela Universidade Federal de Campina Grande. Adrianus Cornelius van Haandel - Professor do Departamento de Engenharia Civil da Universidade Federal de Campina Grande. Heraldo Antunes Silva Filho - Professor do Curso de Saneamento Ambiental do Instituto Federal de Educação Ciência e Tecnologia do Ceará (IFCE).

Geilza Alves Porto - Mestranda em Tecnologia de Polímeros no Instituto de Macromoléculas pela Universidade Federal do Rio de Janeiro. *Endereço para correspondência: luizguilhermeacmagmail.com.
} 


\section{INTRODUÇÃO}

Em sistemas de tratamento com sólidos em suspensão, como o lodo ativado, a sedimentabilidade é utilizada para indicar a eficiência na clarificação da água do efluente e do adensamento dos sólidos no final do processo. A sua medição é adotada para fins de projeto e de monitoramento de desempenho, tendo por objetivo a otimização do processo de clarificação do efluente e a prevenção de problemas com a sedimentabilidade do lodo. Nesse sentido, o estudo de métodos confiáveis para medir a sedimentabilidade é importante e necessário para facilitar o diagnóstico da situação do sistema em escala real.

O primeiro método usado para medir a sedimentabilidade do lodo foi proposto por Mohlman (1934) edenominado índice volumétrico de lodo (IVL). A medição consiste em determinar a razão entre o volume ocupado por 1 litro de lodo após 30 minutos de sedimentação tranquila e a concentração inicial de sólidos suspensos do lodo avaliado. Trata-se de um teste simples e com utilização prática no monitoramento de lodos de sistemas de tratamento. Entretanto, os seus resultados são influenciados pelo volume e concentração inicial de sólidos em suspensão da amostra utilizada (MARAIS e VAN HAANDEL, 1999). Por essa razão, o valor numérico da IVL não dá informação clara sobre a sedimentabilidade do lodo.

Alguns pesquisadores buscaram alternativas para aperfeiçoar o método IVL. Stobbe (1964) desenvolveu o índice volumétrico de lodo diluído (IVLD), estabelecendo que o volume final de lodo deve ser menor do que $200 \mathrm{ml}$ para que o resultado não seja influenciado pela concentração inicial. White (1975) desenvolveu o índice volumétrico do lodo agitador (IVLA), que estabelece uma relação entre o volume de lodo e a unidade de massa de sólidos suspensos em ensaios com uma proveta de 1 litro após 30 minutos de sedimentação mediante uma agitação mecânica suave. Esses aprimoramentos reduziram, porém não eliminaram o problema do IVL.

Vesilind (1968) propôs quantificar a sedimentabilidade de lodo como uma função entre a velocidade de sedimentação em zona e a sua concentração de lodo, em que duas grandezas mecânicas, denominadas constantes $\mathrm{k} \mathrm{e} \mathrm{v}_{0}$, exercem influencia no comportamento da sedimentação, conforme a Equação 1. A constante $k$ está relacionada à compressibilidade do lodo (quanto menor o valor de k, maior é o adensamento do lodo em um determinado volume) e a constante $v_{0}$ representa a velocidade de sedimentação inicial com uma diluição com concentração de lodo tal que as partículas se sedimentariam individualmente (sedimentação não estorvada). Ambas as constantes são determinadas mediante ensaios com diferentes diluições de licor misto, reproduzindo resultados que independem de sua concentração inicial.

$v=v_{0} \cdot \exp \left(-k X_{t}\right)$

Onde:

$v=$ velocidade de sedimentação em zona $\left(m \cdot h^{-1}\right)$

$\mathrm{k}=$ constante de sedimentabilidade ligada à compressibilidade do lodo (l.g-1)

$\mathrm{v}_{0}=$ constante de sedimentabilidade ligada à velocidade de sedimentação $\left(\mathrm{m} \cdot \mathrm{h}^{-1}\right)$

Estudos realizados por Consentino et al. (2005), Figueiredo et al. (2006) e Sales (2013) vêm demonstrando a validade da equação de Vesilind para sistemas de tratamento. Nesses trabalhos, observa-se que a sua aplicação prática vai além da medição convencional em sistemas de lodo ativado, podendo adotá-lo, também, na caracterização de lodos anaeróbios floculentos de reatores UASB para tratamento de esgoto e até mesmo de lodo anaeróbio granulado para tratamento de vinhoto. Portanto, a sua adoção como metodologia para determinar a sedimentabilidade de lodos se torna 
válida e relevante para diferentes sistemas tratamento biológico com suspensão de sólidos.

O método estático, proposto por White (1975) e posteriormente melhorado por Van Haandel e Marais (1999), é o método mais empregado para a determinação das constantes de Vesilind. Os ensaios são feitos em colunas de sedimentação transparentes e milimetradas, onde é observado o comportamento da interface lodo/sobrenadante de amostras de licor misto com diferentes concentrações de lodo em função do tempo, mediante uma leve agitação. Assim, gráficos podem ser feitos para determinar o perfil da sedimentação zonal em função da concentração de lodo $(\mathrm{X})$, tornando possível caracterizar a velocidade de sedimentação do lodo (v), que corresponde à parte linear da curva do gráfico. Correlacionando os valores logarítmicos da velocidade de sedimentação com as suas respectivas concentrações diluídas, obtém-se um gráfico linear que satisfaz a Equação 2, onde a constante $k$ é a declividade da reta e a constante $v_{0}$ é a intersecção da reta com a ordenada.

$\ln (v)=\ln \cdot v_{0} \cdot\left(-k X_{t}\right)$

Embora seja um ensaio experimental confiável para determinar as constantes de Vesilind, o método estático possui desvantagens que limitam o seu uso na rotina de monitoramento da sedimentabilidade do lodo. 0 ensaio normalmente é demorado e demanda um acompanhamento constante do laboratorista para anotação dos dados, tornando-o pouco prático frente a outros métodos menos confiáveis. Por essa razão, seu uso não é tão frequente quanto o método IVL.

O método dinâmico foi desenvolvido no laboratório da EXTRABES, Campina Grande - PB, com o objetivo de automatizar a rotina experimental observada no método estático. Em uma coluna de sedimentação milimetrada, uma amostra de licor misto com concentração de lodo conhecida $\left(X_{\mathrm{t}}\right)$ é submetida à expansão ascensional causa- da por uma força de arraste aplicada por vazões líquidas de uma bomba hidráulica. Quando a interface sobrenadante/lodo se estabiliza em uma determinada altura $(\mathrm{H})$, admite-se que houve um equilíbrio entre a velocidade de sedimentação do lodo ( $v$ ) e a velocidade ascendente do líquido $\left(v_{1}\right)$, conforme a Equação 3 . A concentração de lodo $X_{t}$ de cada valor $v$ pode ser estimada pela razão entre a concentração de sólidos da amostra de lodo (M) e o volume de lodo expandido ( $\left.V_{1}\right)$, conforme a Equação 4. Dessa forma, determinam-se os dados necessários para calcular as constantes de Vesilind, satisfazendo a equação 1.

$v_{1}=Q / A=v=v_{0} \operatorname{Exp}\left(-k X_{t}\right)$

Onde:

$V_{1}=$ velocidade ascendente do líquido $(m \cdot h-1)$

Q = vazão de água aplicada (l.h-1)

$\mathrm{A}=$ área da coluna de sedimentação $\left(\mathrm{m}^{2}\right)$

$X_{t}=M / V_{1}=M /(H . A)$

Onde:

$M=$ massa de lodo na coluna de sedimentação $(\mathrm{g})$.

$V_{1}=$ volume do lodo quando se estabelece o equilíbrio (L)

$\mathrm{H}=$ altura da interface sobrenadante/lodo $(\mathrm{cm})$

Naturalmente, espera-se que os valores obtidos com o método dinâmico sejam iguais àqueles obtidos com o método estático, uma vez que se baseiam na mesma equação básica de Vesilind. Dessa forma, realizou-se uma investigação experimental para comparar resultados dos métodos estático e dinâmico de determinação das constantes $\mathrm{k}$ e $v_{0}$ e verificar a validade da Equação de Vesilind para caracterização da sedimentabilidade de diferentes sistemas de lodo ativado. 


\section{MATERIAIS E MÉTODOS}

A investigação foi feita com lodo obtido de 3 sistemas de lodo ativado em escala de bancada no laboratório da EXTRABES da UEPB, operados em temperatura de $25 \pm 1^{\circ} \mathrm{C}$ e alimentados com esgoto doméstico pré-gradeado da cidade de Campina Grande - PB por um período de 155 dias.

Foram operados três sistemas de lodo ativado distintos: Sistema UCT (Figura 1), Sistema RBS unitário para nitrificação e desnitrificação em um único reator (Figura 2) e Sistema RBS de câmaras dedicadas (Figura 3), com ambientes para nitrificação e desnitrificação em reatores distintos. Os parâmetros de projeto, tais como volume, tempo de detenção hidráulica (TDH), carga orgânica e a concentração média de sólidos totais (SST) do lodo de cada sistema podem ser observados na Tabela 1. A idade de lodo adotada para todos os sistemas foi de 20 dias, tendo por objetivo produzir lodo com bactérias nitrificantes.

Tabela 1: Valores de volume (V), tempo de detenção hidráulica (TDH), carga orgânica e concentração média de sólidos suspensos totais (SST) do lodo durante o período experimental dos sistemas de lodo ativado.

\begin{tabular}{|l|c|c|c|c|}
\hline Sistemas & $\begin{array}{c}\text { V } \\
\text { (I) }\end{array}$ & $\begin{array}{c}\text { TDH } \\
\text { (h) }\end{array}$ & $\begin{array}{c}\text { Carga } \\
\text { Orgânica } \\
\left(\mathbf{g . l}^{-1} \mathbf{- 1}\right)\end{array}$ & $\begin{array}{c}\text { SST } \\
\left(\mathbf{g} . \mathbf{I}^{-\mathbf{1}} \mathbf{)}\right.\end{array}$ \\
\hline UCT & 240 & 20 & 216 & 3,8 \\
\hline RBS Unitário & 15 & 9 & 28,8 & 3,5 \\
\hline $\begin{array}{l}\text { RBS Câmaras } \\
\text { Dedicadas }\end{array}$ & 30 & 4,8 & 57,6 & 3,5 \\
\hline
\end{tabular}

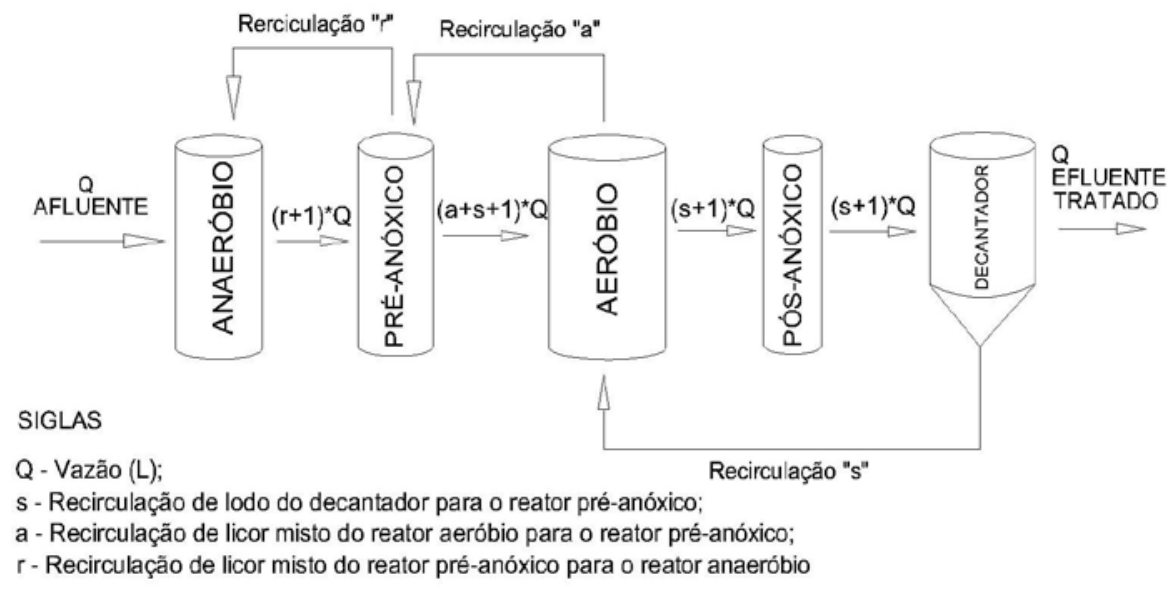

Figura 1: Representação esquemática do sistema UCT.

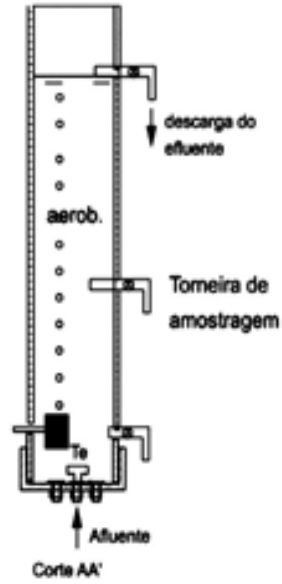

Figura 2: Representação esquemática do sistema RBS unitário.

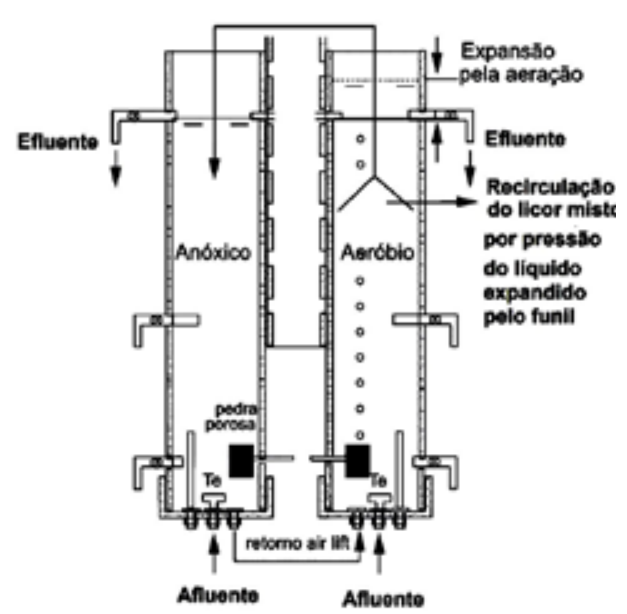

Figura 3: Representação esquemática do sistema RBS com câmaras dedicadas. 


\subsection{Coleta e Tratamento de Amostras}

As amostras de lodo foram coletadas após o descarte de lodo em excesso de cada sistema. Elas foram submetidas à sedimentação por aproximadamente 30 minutos e, em seguida, retirou-se o efluente sobrenadante, sobrando apenas o lodo adensado. Isso foi feito para aumentar a concentração inicial dos sólidos suspensos do lodo, permitindo, assim, um maior alcance de valores $X_{t}$ das bateladas diluídas em ambos os métodos. Uma fração desse lodo adensado foi separada para a realização do método estático, e a outra para realização do método dinâmico.

\subsection{Método Estático}

O método realizado neste trabalho foi uma adaptação da metodologia sugerida por White (1975) e Marais e Van Haandel (1999) conforme a Figura 4. Montou-se uma coluna de sedimentação em acrílico transparente, com volume de 2 litros, altura de $1 \mathrm{~m}$ e diâmetro interno de $5 \mathrm{~cm}$, com uma fita milimétrica no centro da coluna. Um agitador de baixa rotação (4/5 rpm) foi adaptado ao equipamento para promover a sedimentação homogênea do lodo.

Para realizar o ensaio experimental, uma batelada com amostra de lodo adensado com volume de 2 litros foi adicionada suavemente até atingir a altura limite da coluna. Em seguida, procedeu-se a medição da altura da interface sobrenadante/ lodo sedimentado em intervalos de 1 em 1 minuto, totalizando 30 minutos de observações. Ao fim da primeira medição, o conteúdo da batelada foi diluído em uma proporção de $3 / 4$ de lodo por $1 / 4$ de efluente pós-tratado, formando uma nova batelada de lodo diluída para uma nova medição. Para cada ensaio, esse processo foi repetido mais 3 vezes, totalizando 4 bateladas de lodo com concentrações distintas.

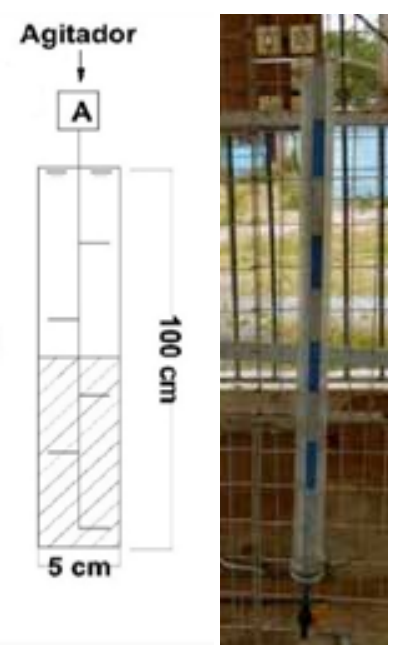

Figura 4: Representação esquemática e visual das colunas de sedimentação utilizados no método estático.

As constantes de Vesilind $\mathrm{ke} \mathrm{v}_{0}$ de cada teste foram determinadas por meio da identificação da velocidade de sedimentação zonal ( $v$ ) e a concentração de sólidos totais $\left(X_{t}\right)$ para satisfazer a Equação 1.0 valor $v$ de cada batelada foi determinado pela identificação da inclinação da reta tangente do deslocamento da interface sobrenadante/lodo em função do tempo e o valor de $X_{t}$ foi obtida em análises laboratoriais. Plotou-se, então, um diagrama semilogarítimico correlacionando os valores logarítmicos ( $I n$ ) de $v$ com os valores de $X_{t}$ para determinar a constante $k$ ( $a$ inclinação da reta) e $v_{0}$ (a intersecção da reta com a ordenada).

\subsection{Método Dinâmico}

O método utilizado foi realizado conforme a metodologia proposta por Leitão et al 2004. Foi utilizada uma coluna de sedimentação construída em acrílico transparente, com volume de 2 litros, altura de $1 \mathrm{~m}$ e diâmetro interno de $5 \mathrm{~cm}$ e munida com uma fita milimétrica no centro da coluna, para determinar a altura de deslocamento sobrenadante/lodo. A coluna foi equipada com uma bomba de recirculação e um agitador de baixa rotação (4/5 rpm), conforme a Figura 5. 
Para realizar o ensaio experimental, utilizou-se um volume de amostra de lodo adensado equivalente a 1/3 do volume da coluna de sedimentação. Em seguida, foi determinada a massa de lodo $(M)$ da amostra antes do início do ensaio experimental, para satisfazer os parâmetros de cálculo da Equação 4. Parte da coluna foi preenchida com a amostra de lodo, e o restante com efluente, até atingir válvula de saída em sua superfície para a recirculação líquida pela bomba. Com a ajuda de uma bomba dosadora, aplicaram-se 5 diferentes vazões (Q) líquidas com velocidades de ascensão conhecidas para a expansão o lodo. Quando a velocidade ascensional líquida aplicada se equilibrava com a velocidade de sedimentação zonal (v), foi anotada a altura da interface sólido-líquido $(\mathrm{H})$. As medições foram utilizadas para estimar as velocidades de sedimentação zonal do lodo ( $v$ ) e as concentrações de lodo $\left(\mathrm{X}_{\mathrm{t}}\right)$ do volume de lodo expandido.
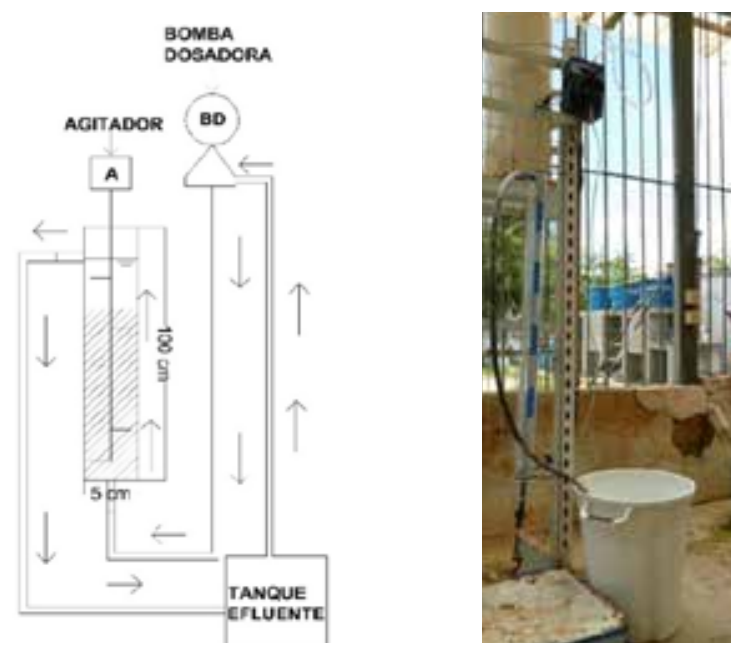

Figura 5: Representação esquemática e visual da coluna de sedimentação utilizado no método dinâmico.

A velocidade de sedimentação zonal ( $v$ ) foi calculada, utilizando-se a Equação 3, sendo a razão entre a vazão de efluente aplicado para a expansão de lodo e a área de lodo expandido após a sua estabilidade. $A$ concentração de lodo $\left(X_{\mathrm{t}}\right)$ de cada medição foi calculada por meio da Equação 4, que é a razão entre a concentração de lodo da amostra (M) e o volume de lodo expandido quando se estabelece o equilíbrio $\left(V_{1}\right)$. Os valores de $v$ e $X_{t}$ das diferentes medições foram plotados em diagramas semilogarítimicos para determinar as constantes de Vesilind, da mesma forma que no método estático.

\subsection{Avaliação dos dados}

Os resultados obtidos nos dois ensaios foram submetidos à análise estatística descritiva, tais como a média, o desvio padrão, o coeficiente de variação (CV) e os máximos e mínimos. Em seguida, realizou-se a análise estatística Tukey em nível de probabilidade de $5 \%$, para verificar se houve diferença entre as médias avaliadas. 0 software adotado para essa análise foi o SISVAR versão 5.3.

A validade da equação de Vesilind foi determinada a partir do cálculo da concentração $X_{t}$ em uma velocidade de sedimentação factível de $1 \mathrm{~m} \cdot \mathrm{h}^{-1}$, adotando-se a Equação 1 e as constantes dos valores mínimos, médios e máximos determinados pelos dois métodos em estudo.

\section{RESULTADOS E DISCUSSÕES}

Os resultados experimentais obtidos pelo método estático para determinar as constantes de Vesilind dos três sistemas de lodo ativado são apresentados nas Figuras 6, 7 e 8. Nos diagramas (a) de cada figura estão expressos o comportamento da sedimentação das bateladas diluídas de lodo $\left(\mathrm{X}_{\mathrm{t}}\right)$ em função do tempo. Já nos diagramas (b) estão as correlações semilogarítmicas entre (v) e a concentração do lodo $X_{t}$.

As Figuras 6, 7 e 8 representam o comportamento típico dos sete ensaios de cada um dos sistemas de lodo ativado em estudo. Nos diagramas (a), cada batelada de lodo com concentração $X_{t}$ apresentou um comportamento de desloca- 
mento de interface sobrenadante/lodo distinto. Considerando que a velocidade de sedimentação corresponde à inclinação da reta tangente do gráfico, quanto mais diluída a batelada de lodo, maior o valor de v. O valor do coeficiente $r^{2}$ apresentado na correlação semilogarítmica dos diagramas (b) ficou entre 0,985 e 0,995, constatando a boa correlação entre os dados experimentais para determinar as constantes $\mathrm{k} \mathrm{e}_{0}$ de Vesilind.
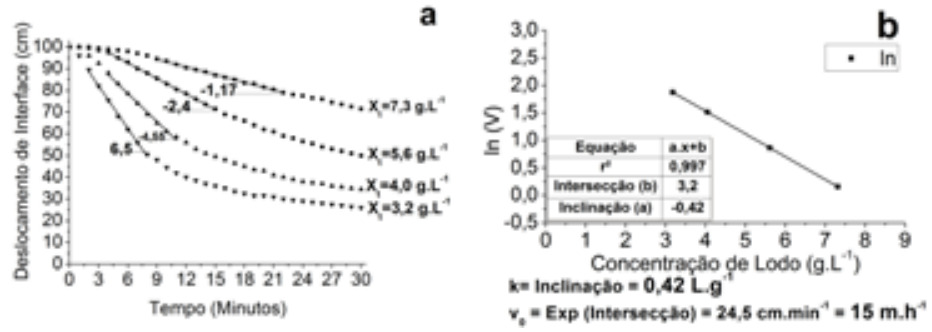

Figura 6: Perfil do deslocamento da interface sobrenadante/lodo em função do tempo (a) e do diagrama semilogarítmico (b) do método estático para o reator RBS unitário.
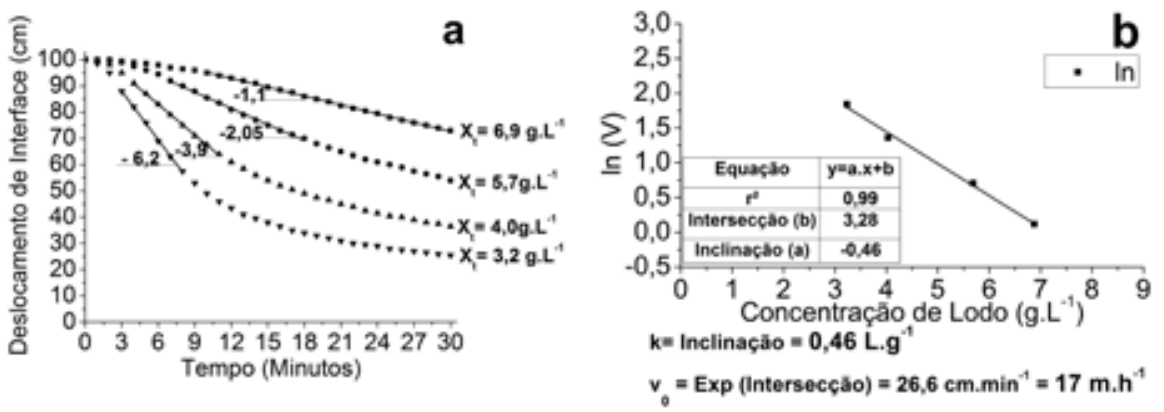

Figura 7: Perfil do deslocamento da interface sobrenadante/lodo em função do tempo (a) e do diagrama semilogarítmico (b) do método estático para o reator RBS câmaras dedicadas.
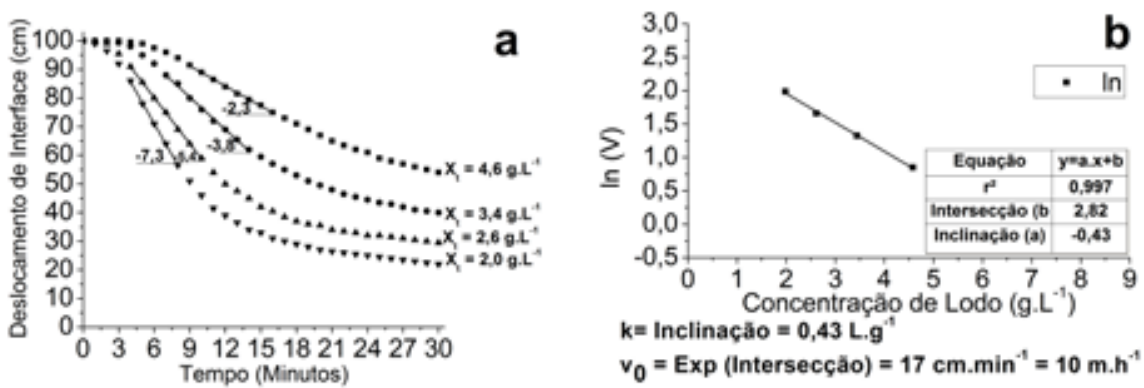

Figura 8: Perfil do deslocamento da interface sobrenadante/lodo em função do tempo (a) e do diagrama semilogarítmico (b) do método estático para o sistema UCT. 
Os resultados experimentais obtidos pelo método dinâmico para determinar as constantes de Vesilind dos três sistemas de lodo ativado estão apresentados na Tabela 2 (resultados experimentais para cálculo de $v$ e $X_{\mathrm{t}}$ ) e na Figura 9 (correlação semilogarítmica). Observa-se que os parâmetros vazão (Q) e altura de equilíbrio sobrenadante/lodo $(\mathrm{H})$ influenciam nos cálculos de determinação de $v$ e $\mathrm{X}_{\mathrm{t}}$, tendo em vista que eles estão associados à velocidade de arraste do lodo e à expansão de volume $\left(V_{1}\right)$. Aplicando os valores calculados de $v$ e $X_{t}$ no diagrama semilogarítimico da Figura 9, foi possível obter correlações com $r^{2}$ próximo a 0,99 em todos os sete testes realizados.

Tabela 2: Resultados experimentais do método dinâmico dos sistemas RBS Unitário (a), RBS Câmaras Dedicadas (b) e sistema UCT (c).

\begin{tabular}{|c|c|c|c|c|c|}
\hline $\mathbf{I}_{\mathbf{a}^{-1}}$ & $\begin{array}{c}\text { V } \\
m \cdot h^{-1}\end{array}$ & $\begin{array}{c}\mathrm{H} \\
\mathrm{Cm}\end{array}$ & $\begin{array}{l}\text { VI } \\
\text { I }\end{array}$ & $\underset{\mathrm{g} \cdot \mathrm{I}^{-1}}{\mathrm{x}^{-}}$ & $\begin{array}{c}\ln (v) \\
-\end{array}$ \\
\hline \multicolumn{6}{|c|}{ RBS Unitário } \\
\hline 2,7 & 1,4 & 60 & 1,18 & 5,14 & 0,32 \\
\hline 3,6 & 1,8 & 67 & 1,32 & 4,60 & 0,61 \\
\hline 4,5 & 2,3 & 78,5 & 1,54 & 3,93 & 0,83 \\
\hline 5,4 & 2,8 & 84 & 1,65 & 3,67 & 1,02 \\
\hline 6,3 & 3,2 & 93 & 1,83 & 3,32 & 1,17 \\
\hline \multicolumn{6}{|c|}{ RBS Câmaras Dedicadas } \\
\hline 2,7 & 1,4 & 47,5 & 0,93 & 5,43 & 0,32 \\
\hline 3,6 & 1,8 & 54 & 1,06 & 4,77 & 0,61 \\
\hline 5,4 & 2,8 & 68 & 1,33 & 3,79 & 1,02 \\
\hline 6,9 & 3,5 & 78,5 & 1,53 & 3,28 & 1,26 \\
\hline 8,4 & 4,3 & 86 & 1,68 & 3,00 & 1,46 \\
\hline \multicolumn{6}{|c|}{ UCT } \\
\hline 2,7 & 1,4 & 34,00 & 0,67 & 4,99 & 0,32 \\
\hline 3,6 & 1,8 & 39,50 & 0,77 & 4,30 & 0,61 \\
\hline 5,4 & 2,8 & 52,50 & 1,03 & 3,23 & 1,02 \\
\hline 7,2 & 3,7 & 61,00 & 1,19 & 2,78 & 1,30 \\
\hline 8,1 & 4,1 & 68,00 & 1,33 & 2,50 & 1,42 \\
\hline
\end{tabular}
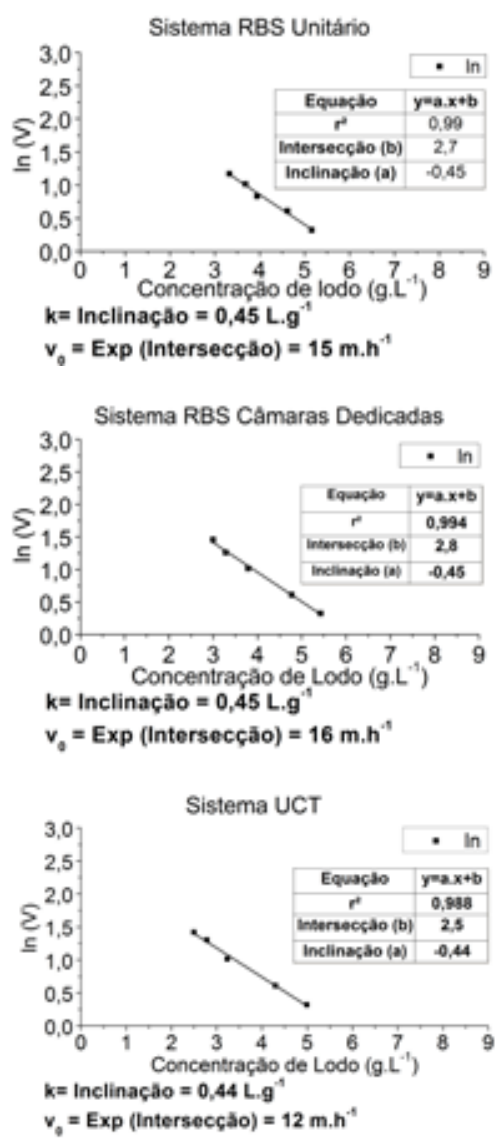

Figura 9: Diagrama de correlação semilogarítmica da velocidade de sedimentação em função da concentração de lodo para os três sistemas de lodo ativado.

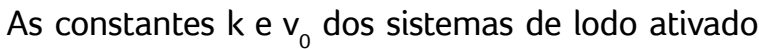
RBS Unitário, RBS Câmaras Dedicadas e fluxo contínuo UCT obtidas pelos ensaios do método estático e dinâmico, bem como o tratamento estatístico para a sua avaliação podem ser observados na Tabela 3. Os dados de k estão expressos em l.g-1 $\mathrm{v}_{0}$ em m.h $\mathrm{h}^{-1}$, e os valores estatísticos descritivos e de comparação de médias descritos no final da tabela. 0 resultado do teste Tukey em nível de significância de 5\% para cada sistema de lodo ativado é caracterizado pela letras ao lado dos valores médios, onde a letra maiúscula representa a comparação das médias da constante $k$ e as minúsculas a constante $v_{0}$. 
$\mathrm{Na}$ estatística descritiva, os valores médios da compressibilidade do lodo ( $k$ ) ficaram entre 0,43 e $0,45 \mathrm{~m} \cdot \mathrm{h}^{-1}$ e da velocidade inicial da sedimentabilidade $\left(v_{0}\right)$ entre 11 e $19 \mathrm{~m} \cdot \mathrm{h}^{-1}$. O maior coeficiente de variação (CV) da constante $\mathrm{k}$ foi encontrado nos resultados do método dinâmico, com 13\% nos reatores RBS unitário e RBS de câmaras dedicadas. $\mathrm{Na}$ constante $\mathrm{v}_{0}$, os maiores valores de $\mathrm{CV}$ foram encontrados no método estático, com variações de $15 \%$ e $19 \%$ também nos sistemas RBS unitário e RBS câmaras dedicadas. Ressalta-se que o coeficiente de variação é calculado por meio da relação entre o desvio padrão e o valor médio, correspondendo ao percentual de variação dos resultados experimentais em relação a sua média.

$\mathrm{Na}$ análise estatística Tukey em nível de probabilidade de $5 \%$, observa-se que as médias das constantes $\mathrm{k}$ e $\mathrm{v}_{0}$ não apresentaram diferenças significativas entre si, apresentando as mesmas letras em todas as comparações. Apesar dos di- ferentes valores do coeficiente de variação, houve uma similaridade estatística na reprodução de resultados dos dois ensaios experimentais, demonstrando que ambos os métodos foram capazes de expressar as mesmas características de sedimentabilidade de lodo dos sistemas de tratamento em estudo.

Ao comparar os resultados reproduzidos por ambos os métodos com a literatura, observa-se que os sistemas obtiveram comportamento insatisfatório em termos de compressibilidade. Marais \& Van Haandel (1999) estabelecem que valores próximos ou maiores que $0,45 \mathrm{l}^{-g^{-1}}$ são considerados ruins para a operação de sistemas de lodo, implicando em alterações na operação dos decantadores para não haver descarga de lodo junto com o efluente. Isso reforça a importância do monitoramento da qualidade do lodo dos sistemas de tratamento pelas constantes de Vesilind, sendo uma ferramenta necessária para a tomada de decisão.

Tabela 3: Valores das constantes $k$, $v_{0}$ calculados pela equação de Vesilind dos sistemas de lodo ativado em função dos métodos estático e dinâmico e tratamento estatístico pelo método Tukey.

\begin{tabular}{|c|c|c|c|c|c|c|c|c|c|c|c|c|}
\hline \multirow{3}{*}{ Testes } & \multicolumn{4}{|c|}{ RBS Unitário } & \multicolumn{4}{|c|}{ RBS Câmaras Dedicadas } & \multicolumn{4}{|c|}{ Sistema UCT } \\
\hline & \multicolumn{2}{|c|}{$k\left(l . g^{-1}\right)$} & \multicolumn{2}{|c|}{$v_{0}\left(m \cdot h^{-1}\right)$} & \multicolumn{2}{|c|}{$k\left(1 . g^{-1}\right)$} & \multicolumn{2}{|c|}{$v_{0}\left(m \cdot h^{-1}\right)$} & \multicolumn{2}{|c|}{$k\left(l . g^{-1}\right)$} & \multicolumn{2}{|c|}{$v_{0}\left(m \cdot h^{-1}\right)$} \\
\hline & Est. & Din. & Est. & Din. & Est. & Din. & Est. & Din. & Est. & Din. & Est. & Din. \\
\hline 1 & 0,42 & 0,33 & 15 & 14 & 0,42 & 0,37 & 10 & 20 & 0,44 & 0,32 & 10 & 13 \\
\hline 2 & 0,42 & 0,49 & 14 & 13 & 0,41 & 0,4 & 15 & 19 & 0,43 & 0,47 & 11 & 12 \\
\hline 3 & 0,4 & 0,49 & 14 & 16 & 0,5 & 0,45 & 18 & 19 & 0,42 & 0,45 & 11 & 11 \\
\hline 4 & 0,43 & 0,45 & 15 & 14 & 0,46 & 0,34 & 16 & 16 & 0,46 & 0,41 & 14 & 11 \\
\hline 5 & 0,44 & 0,49 & 13 & 16 & 0,48 & 0,44 & 19 & 19 & 0,43 & 0,5 & 11 & 14 \\
\hline 6 & 0,42 & 0,45 & 14 & 13 & 0,49 & 0,5 & 19 & 17 & 0,44 & 0,49 & 11 & 13 \\
\hline 7 & 0,46 & 0,39 & 14 & 20 & 0,39 & 0,5 & 14 & 17 & 0,5 & 0,5 & 12 & 14 \\
\hline Média & $0,43 A$ & $0,44 A$ & $14 a$ & $15 a$ & $0,45 A$ & $0,43 A$ & $16 a$ & $18 a$ & $0,45 A$ & $0,45 A$ & $11 a$ & $13 a$ \\
\hline DP & 0,03 & 0,06 & 0,64 & 2,29 & 0,04 & 0,06 & 3,00 & 1,36 & 0,02 & 0,06 & 1,18 & 1,18 \\
\hline CV & $4 \%$ & $13 \%$ & $5 \%$ & $15 \%$ & $9 \%$ & $13 \%$ & $19 \%$ & 7\% & $6 \%$ & $13 \%$ & $10 \%$ & $9 \%$ \\
\hline Máx & 0,46 & 0,49 & 15 & 20 & 0,5 & 0,5 & 19 & 20 & 0,5 & 0,5 & 14 & 14 \\
\hline Mín & 0,4 & 0,33 & 13 & 13 & 0,39 & 0,34 & 10 & 16 & 0,42 & 0,32 & 10 & 11 \\
\hline
\end{tabular}

Obs.: Médias seguidas da mesma letra não possuem diferenças significativas entre si em nível de probabilidade de $5 \%$. Para cada sistema de lodo ativado, as letras maiúsculas comparam médias da constante $\mathrm{k}$ e as letras minúsculas comparam médias da constante $\mathrm{v}_{0}$ dos métodos estático e dinâmico. 
$\mathrm{Na}$ tabela 4 estão expressos os valores calculados da concentração de lodo $X_{t}$ para uma velocidade de sedimentação (v) fixa de $1 \mathrm{~m} \cdot \mathrm{h}^{-1}$, aplicando os valores mínimos, médios e máximos das constantes $\mathrm{k}$ e $\mathrm{v}_{0}$ da Tabela 3 na Equação 1. Os números mostram que é possível estimar a concentração de lodo sedimentado $\left(X_{t}\right)$ para valores fixos de velocidade de sedimentação ( $v$ ) quando as constantes $k$ e $v_{0}$ são adotadas como parâmetro. Considerando que o volume de lodo é inversamente proporcional à sua concentração, as constantes de Vesilind são preponderantes para estimar o volume de lodo ocupado em tanques de tratamento, tendo influência, portanto, sobre os custos de projeto e controle operacional. Esses dados estão de acordo com os de Consentino et al. (2005), Figueiredo et al. (2006) e Sales (2013), que verificaram o mesmo comportamento em seus experimentos.

Tabela 4: Valores calculados de $X_{t}$ em função das constantes $k$ e $v_{0}$ dos sistemas de lodo ativado para $v=1 \mathrm{~m} \cdot \mathrm{h}^{-1}$.

\begin{tabular}{|c|c|c|c|}
\hline \multicolumn{2}{|l|}{ Sistemas } & $\begin{array}{l}\text { Método } \\
\text { Estático }\end{array}$ & $\begin{array}{l}\text { Método } \\
\text { Dinâmico }\end{array}$ \\
\hline \multirow{4}{*}{ RBS UNITÁRIO } & \multicolumn{3}{|c|}{ Xt $\left(g . l^{-1}\right)$ para v = $1 \mathrm{~m} \cdot \mathrm{h}^{-1}$} \\
\hline & Mín & 6,41 & 7,75 \\
\hline & Média & 6,14 & 6,14 \\
\hline & Máx & 6,13 & 6,1 \\
\hline \multirow{3}{*}{$\begin{array}{l}\text { RBS CAMARAS } \\
\text { DEDICADAS }\end{array}$} & Mín & 5,9 & 8,15 \\
\hline & Média & 6,14 & 6,7 \\
\hline & Máx & 5,89 & 5,99 \\
\hline \multirow{3}{*}{ UCT } & Mín & 5,48 & 7,49 \\
\hline & Média & 5,32 & 5,68 \\
\hline & Máx & 5,26 & 5,26 \\
\hline
\end{tabular}

\section{CONSIDERAÇÕES FINAIS}

Nos ensaios experimentais realizados neste trabaIho foi possível constatar que os métodos estático e dinâmico deram conclusões equivalentes sobre as constantes de Vesilind, tendo em vista a análise estatística Tukey em nível de probabilidade de 5\%. Apesar disso, é interessante a realização de outros estudos comparativos para dar maior validade às conclusões obtidas nesta pesquisa. Isso poderia ser feito por meio de experimentos com uma maior quantidade de repetições, para melhorar qualidade dos resultados estatísticos.

Outro aspecto importante é a confirmação da equação de Vesilind como metodologia válida para caracterizar a sedimentabilidade do lodo em sistemas de lodo ativado, tendo em vista a sua capacidade de estimar a qualidade do lodo dos sistemas de fluxo contínuo (UCT) e de sistemas RBS para diferentes concentrações $X_{t}$. Esses resultados confirmam a eficácia desse método na medição da sedimentabilidade do lodo, reforçando a importância do seu uso no projeto e monitoramento em sistemas de tratamento de lodo ativado.

\section{REFERÊNCIAS}

BARBOSA, C. A. C. e SOUZA, M. A. A. Sedimentabilidade do lodo secundário obtido no processo de lodos ativados com remoção biológica de nutrientes. In: Asociación Peruana de Ingenieria Sanitaria y Ambiental; AIDIS. Gestión ambiental en el siglo XXI. Lima-Peru, 1998.

CATUNDA P.F.C., SOUZA J. T. E VAN HAANDEL A.C. Determinação da sedimentabilidade de lodo ativado. In: $14^{\circ}$ Congresso Brasileiro de Engenharia Sanitária e ambiental, Belém - PA, 1989.

CONSENTINO, P. R. S.; FILHO, J. A. S.; VAN HAANDEL; CATUNDA, P. F. F. C. Estabilização de lodo de sistemas aeróbios em reatores do tipo UASB. In: $23^{\circ}$ Congresso Brasileiro de Engenharia Sanitária e ambiental, Campo Grande - Mato Grosso do Sul, ABES CD-ROM, 2005.

FIGUEREDO, G.P.; VAN HAANDEL, A.C.; SILVA FILHO, J. A; PAIVA, F.V. Cavalcanti, P.F.F. Determinação da sedimentabilidade de lodo de sistemas anaeróbio-aeróbios tratando esgoto. In: Simpósio ítalo Brasileiro de Engenharia Sanitária e Ambiental, Fortaleza-CE ABES CD-ROM. 2006.

LEITÃO, R. C. Robustness of UASB Reactors Treating Sewage Under Tropical Conditions. Tese (Doutorado) - Wageningen University/ Wageningen, 160p. 2004.

MOHLMAN, M. L. The Sludge Index. In: Sew. Works J., 6(1):119, 1934.

SANTOS, V. S. Avaliação da capacidade de produção de metano em sistemas UASB tratando vinhoto sob diferentes condições operacionais. Dissertação (Mestrado). PPGECA, UFCG. 2013

SILVA, A. L.; SILVA FILHO, H. A.; DERKS, Y. M.; CAVALCANTI, P. F. F. C.; VAN HAANDEL, A. C. Determinação das constantes de sedimentabilidade de sistemas de lodo ativado In: XIII SILUBESA - Simpósio 
Luso Brasileiro de Engenharia Sanitária e Ambiental, Belém-PA, ABES CD-ROM, 2008.

STOBBE, G. Über das Verhalten von belebtem Schlamm in aufsteig ender Wasserbewegung. In: Publication of the Sanitation Engineering Institute of the Technical University of Hanover. 18. 100 p. (in German), 1964.
VAN HAANDEL, A. C.; MARAIS, G. V. R. O. Comportamento do Sistema de Lodo Ativado: Teoria e Aplicações para Projetos e Operações. Campina Grande: Epgraf, 72 p, 1999

VESILIND, P. A. Theoretical considerations: Design of prototype thickeners from batch settling tests In: Water and Sewage Works 115:302-307, 1968.

WHITE M. J. D. Settling of activated sludge, technical report TR11, WRC Stevenhage - Reino Unido, 1975. 